\title{
Internalization of mesoporous silica nanoparticles induces transient but not sufficient osteogenic signals in human mesenchymal stem cells
}

\author{
Dong-Ming Huang a,*, Tsai-Hua Chung ${ }^{\mathrm{b}}$, Yann Hung ${ }^{\mathrm{c}}$, Fang Lu ${ }^{\mathrm{c}}, \mathrm{Si}-\mathrm{Han} \mathrm{Wu}^{\mathrm{c}}$, \\ Chung-Yuan Mou ${ }^{\mathrm{c}}$, Ming Yao ${ }^{\mathrm{d}}$, Yao-Chang Chen ${ }^{\mathrm{b}, \mathrm{d}, \mathrm{e}, *}$ \\ a Center for Nanomedicine Research, National Health Research Institutes, Miaoli 350, Taiwan \\ b Stem Cell Research Center, National Health Research Institutes, Miaoli 350, Taiwan \\ c Department of Chemistry, National Taiwan University, Taipei 106, Taiwan \\ d Department of Laboratory Medicine, National Taiwan University Hospital and College of Medicine, National Taiwan University, Taipei 100, Taiwan \\ e Department of Forensic Medicine, National Taiwan University Hospital and College of Medicine, National Taiwan University, Taipei 100, Taiwan
}

\section{A R T I C L E I N F O}

\section{Article history:}

Received 3 January 2008

Revised 14 April 2008

Accepted 16 April 2008

Available online 25 April 2008

\section{Keywords}

Nanoparticles

Mesenchymal stem cells

Osteogenic differentiation

RhoA

Actin cytoskeleton

Cytotoxicity

\begin{abstract}
A B S T R A C T
The biocompatibility of nanoparticles is the prerequisite for their applications in biomedicine but can be misleading due to the absence of criteria for evaluating the safety and toxicity of those nanomaterials. Recent studies indicate that mesoporous silica nanoparticles (MSNs) can easily internalize into human mesenchymal stem cells (hMSCs) without apparent deleterious effects on cellular growth or differentiation, and hence are emerging as an ideal stem cell labeling agent. The objective of this study was to thoroughly investigate the effect of MSNs on osteogenesis induction and to examine their biocompatibility in hMSCs. Uptake of MSNs into hMSCs did not affect the cell viability, proliferation and regular osteogenic differentiation of the cells. However, the internalization of MSNs indeed induced actin polymerization and activated the small GTPbound protein RhoA. The MSN-induced cellular protein responses as believed to cause osteogenesis of hMSCs did not result in promotion of regular osteogenic differentiation as analyzed by cytochemical stain and protein activity assay of alkaline phosphatase (ALP). When the effect of MSNs on ALP gene expression was further examined by reverse transcriptase polymerase chain reaction, MSN-treated hMSCs were shown to have significantly higher mRNA expression than control cells after 1-hour osteogenic induction. The induction of ALP gene expression by MSNs, however, was absent in cells after 1-day incubation with osteogenic differentiation. Together our results show that the internalization of MSNs had a significant effect on the transient protein response and osteogenic signal in hMSCs, thereby suggesting that the effects of nanoparticles on diverse aspects of cellular activities should be carefully evaluated even though the nanoparticles are generally considered as biocompatible at present.
\end{abstract}

(c) 2008 Elsevier Inc. All rights reserved.

\section{Introduction}

With the recent progress of nanotechnology in biomedical applications (Roco, 2003; Sahoo and Labhasetwar, 2003; Wilkinson, 2003), the use of nanomaterials as biomaterials in fundamental and technological developments has received considerable attentions. For instance, nanoparticles are being widely investigated as delivery carriers of labeling molecules, therapeutic drugs, and nucleic acids for cellular imaging/ tracking (Bulte et al., 1993; Larson et al., 2003), drug targeting, and gene delivery (Bellocq et al., 2003), respectively. Although nanotechnology has been defined as being restricted to objects with dimensions in the range of 1-100 nm, structures and objects up to $1000 \mathrm{~nm}$ in size have

\footnotetext{
* Corresponding authors. D.-M. Huang is to be contacted at Fax: +886 3758 447. Y.-C. Chen, Department of Forensic Medicine, National Taiwan University Hospital and College of Medicine, National Taiwan University, Taipei 100, Taiwan. Fax: +886 223218438 .

E-mail addresses: dmhuang@nhri.org.tw (D.-M. Huang), ycchenmd@ntu.edu.tw (Y.-C. Chen).
}

been widely included in nanomedicine. The unique physical and chemical characteristics of nanoparticles and worries about their potential environmental and health impacts (Colvin, 2003; Giles, 2004; Hoet et al., 2004), however, have prompted calls to evaluate their potential toxicity in biomedical applications. Current in vitro studies reveal some key factors by which nanoparticles introduced into cultured cells could influence biological functions or induce cytotoxicity (Derfus et al., 2004; Huang et al., 2004; Gupta and Gupta, 2005; Hussain et al., 2005; Jia et al., 2005; Yin et al., 2005). Determining whether these nanomaterials are toxic, however, depends greatly on diverse parameters, such as the structural properties of particles, their dosage forms, and their intended uses.

Silica is potentially an ideal nanomaterial for biomedical applications because it can be easily surface functionalized for bioconjugation, and is quite biocompatible and resistant to biodegradation in cellular environments. Particularly, mesoporous silica nanoparticles (MSNs), in the form of stable aqueous dispersion, are emerging as a versatile agent for biomedical imaging, drug delivery, and gene therapy (Lai et al., 2003; 
Giri et al., 2005; Huang et al., 2005; Lin et al., 2005; Slowing et al., 2006; Chung et al., 2007). We are developing MSNs as a platform for stem cell tracking. This type of tracking requires consideration of the biological effects of the internalized nanoparticles. Although the two components of using for stem cell labeling (i.e. silica and imaging probes) are not expected to be significantly cytotoxic and a number of studies have indeed indicated that internalized silica nanoparticles are quite biocompatible with stem cells, 3T3-L1 cells, and HeLa cells (Huang et al., 2005; Lin et al., 2005; Slowing et al., 2006; Chung et al., 2007), the

\section{A}

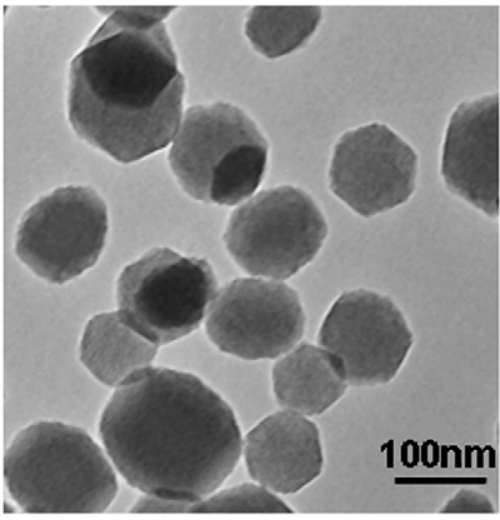

C

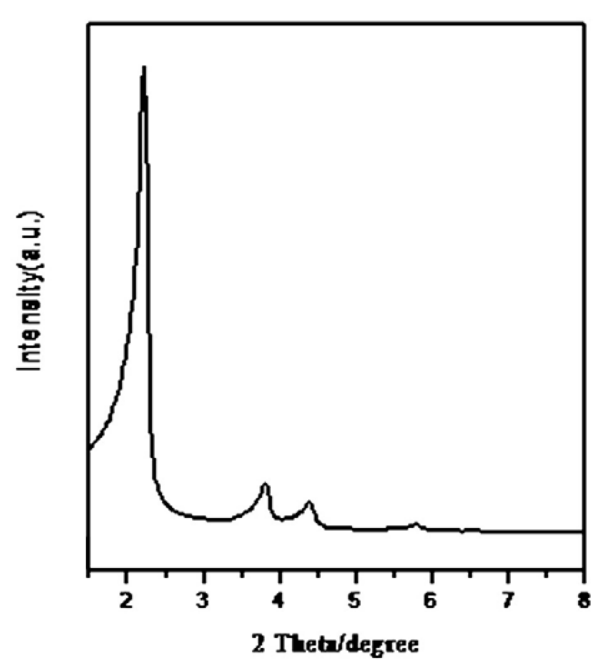

$\mathbf{E}$

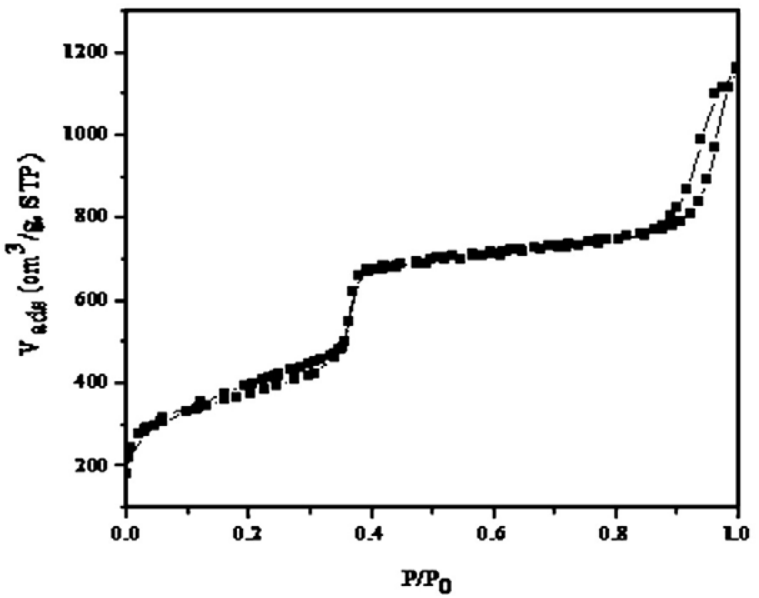

term "biocompatible" can be misleading due to nonexistence of criteria for evaluating the toxic effects of nanomaterials.

In our previous study, we demonstrated that MSNs possess the highly efficient cellular labeling/internalization activity, durability, and, above all, biocompatibility to serve as a nanoparticle carrier for stem cell tracking (Huang et al., 2005; Chung et al., 2007). Our finding that inhibition of actin polymerization by cytochalasin $\mathrm{D}$ decreased the uptake of MSNs, however, suggests a link between actin organization and cellular internalization of MSNs. The dynamic polymerization of
B
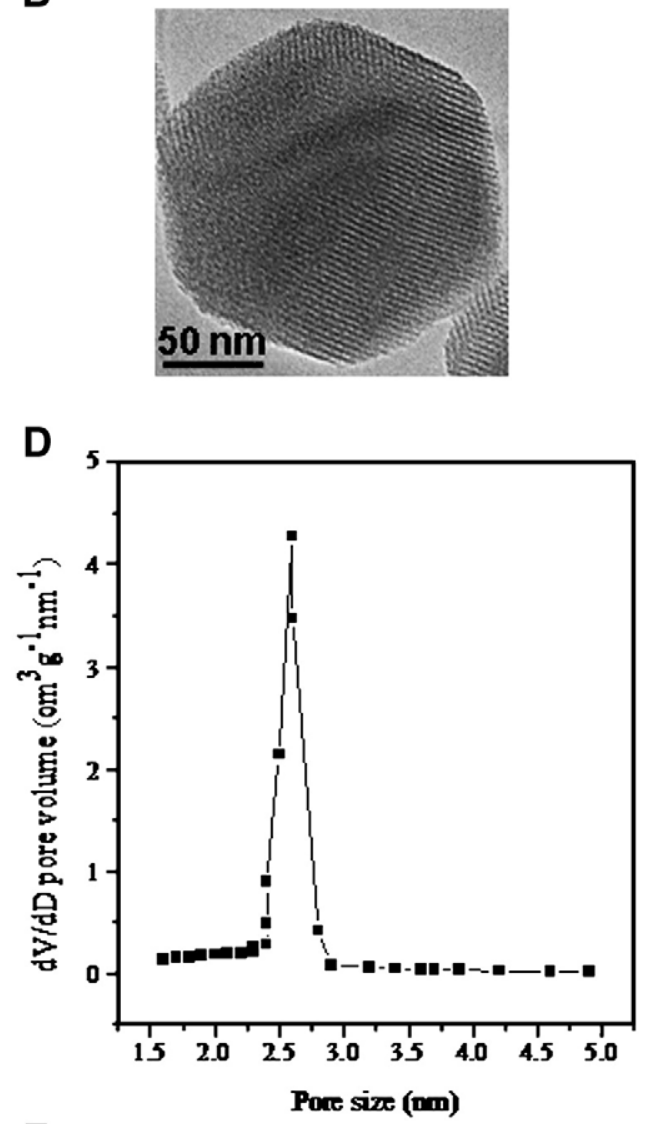

$\mathbf{F}$

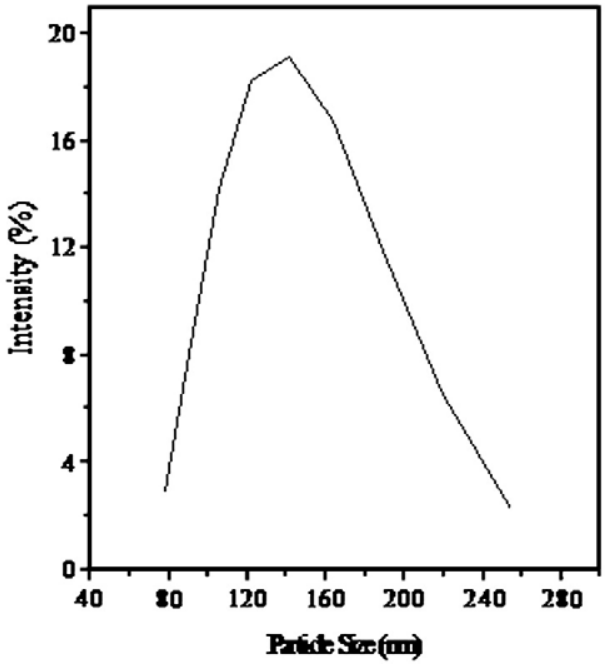

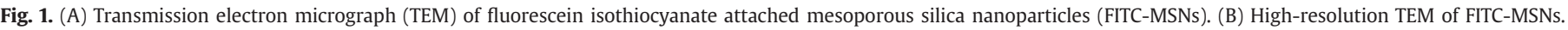

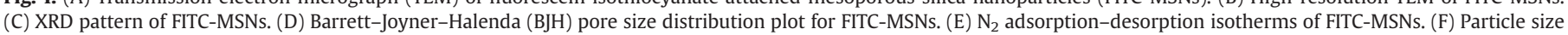
distribution of FITC-MSNs suspended in water and measured by dynamic light scattering (DLS). 
actin filaments has a central role in many important fields. The clear correlation between cell shape and differentiation supports the ideas that changes in cell shape and cytoskeletal integrity are important in differentiation of certain lineages (Ren et al., 1999; Sordella et al., 2003; McBeath et al., 2004; Meyers et al., 2005). Recent studies indicate that the organization of actin cytoskeleton may be critical in supporting the osteogenic differentiation of human mesenchymal stem cells (hMSCs) (McBeath et al., 2004; Meyers et al., 2005). Thus, the internalization of MSNs could likely result in some alterations in cellular proteins responsible for regulating actin dynamics. The effects of uptake of biocompatible nanoparticles on actin organization as well as on the subsequent cellular responses, such as changes in molecular pathway and the process of osteogenesis, have not received much investigation. Therefore, in this study we sought to examine whether the actindependent internalization of MSNs could alter the cytoskeleton organization signal to affect the osteogenic differentiation of hMSCs. All possible cytotoxic effects should be evaluated before a nanomaterial is declared biocompatible; and an awareness of advantages and disadvantages of using "biocompatible" nanomaterials in biomedicine must be fostered.

\section{Materials and methods}

Nanoparticle preparation and characterization. Fluorescein isothiocyanate attached mesoporous silica nanoparticles (FITC-MSNs) with well-ordered hexagonal pore structure were synthesized as described in a previous study (Lin et al., 2005).

Transmission electron microscope (TEM) images were obtained on a JEOL 100CX microscope with a $\mathrm{CeB}_{6}$ filament and an accelerating voltage of $100 \mathrm{kV}$. Samples were prepared by dropping sample suspension onto a carbon-coated, holey film supported on a $3 \mathrm{~mm}, 300$ mesh copper grid. Small-angle powder X-ray diffraction (XRD) patterns were obtained using a Rigaku Rotaflex diffractometer with $\mathrm{Cu} K \alpha$ radiation $(\lambda=0.154 \mathrm{~nm})$. $\mathrm{N}_{2}$ adsorption-desorption isotherms were obtained at $-196 \mathrm{C}$ on a Micromeritics ASAP 2010 sorptometer using static adsorption procedures. Samples were degassed at $100{ }^{\circ} \mathrm{C}$ and $10^{-6}$ Torr for a minimum of $12 \mathrm{~h}$ prior to analysis. The particle size was measured employing Nano ZS90 laser particle analyzer (Malvern Instruments, UK) at $25{ }^{\circ} \mathrm{C}$.

Culture of human mesenchymal stem cells (hMSCs) from bone marrow. hMSCs were isolated from bone marrow of normal donors as described in a previous study (Huang et al., 2005) and were cultured in regular growth medium consisting of low-glucose DMEM (GIBCO) supplemented with 10\% fetal bovine serum (FBS) (GIBCO), $100 \mathrm{U} / \mathrm{ml}$ penicillin, and $100 \mu \mathrm{g} / \mathrm{ml}$ streptomycin. All cultures were kept in an atmosphere of $5 \%$ $\mathrm{CO}_{2}$ and $95 \%$ air at $37{ }^{\circ} \mathrm{C}$.

Cell viability and proliferation assay. In vitro acute cytotoxicity and cell proliferation experiments were assessed using 3-[4,5-dimethylthiazol-2-yl]-2,5-diphenyltetrazolium bromide (MTT) reduction and trypan blue exclusion assays. After incubation with FITC-MSNs suspension in serum-free medium for $1 \mathrm{~h}$, cells were incubated with fresh serum-free medium containing $0.5 \mathrm{mg} / \mathrm{ml}$ MTT for $1 \mathrm{~h}$ at $37^{\circ} \mathrm{C}$ for cytotoxicity assay. For proliferation assay, the cells after treatment with FITC-MSNs for $1 \mathrm{~h}$ were allowed to grow in regular growth medium for $24 \mathrm{~h}$, followed by incubation with MTT reagent or by hemocytometer counts of trypan blue-excluding cells. The dark blue formazan dye generated by the live cells was proportional to the number of live cells; and the absorbance at $570 \mathrm{~nm}$ was measured using a microplate reader. Cell numbers were determined from a standard plot of known cell numbers vs. the corresponding absorbance density.

Flow cytometry detection of FITC-MSNs uptake. hMSCs were seeded at $1.2 \times 10^{5}$ cells per well in six-well plates and allowed to attach for $24 \mathrm{~h}$. To determine the FITC-MSN uptake and loading contents, the cells were incubated with $40 \mu \mathrm{g} / \mathrm{ml}$ of FITC-MSN suspension in serum-free medium for various incubation times. Treated cells were then washed three times with phosphate-buffered saline (PBS: $137 \mathrm{mM} \mathrm{NaCl}, 2.68 \mathrm{mM} \mathrm{KCl}$, $10 \mathrm{mM} \mathrm{Na}_{2} \mathrm{HPO}_{4}, 1.76 \mathrm{mM} \mathrm{KH}_{2} \mathrm{PO}_{4}, \mathrm{pH} 7.4$ ) and then harvested by trypsinization. After centrifugation, the cell pellet was washed once and resuspended with PBS containing $2 \%$ FBS. The fluorescent dye incorporated in FITC-MSNs serves as a marker to semiquantitatively determine their cellular uptake, analyzed by FACSCalibur flow cytometry and CellQuest Pro software (Becton Dickenson, Mississauga, CA).

Immunofluorescence staining of actin cytoskeleton organization. hMSCs plated on glass coverslips were washed with PBS, fixed in $4 \%$ formaldehyde/PBS for $20 \mathrm{~min}$, and permeabilized in $0.1 \%$ Triton X-100 at room temperature for 5 min. Cells were blocked with $1 \%$ BSA for 30 min followed by labeling with rhodamine-phalloidin (Molecular Probes) at a final concentration of $0.2 \mu \mathrm{M}$ in the dark for $30 \mathrm{~min}$. Cells were again washed with PBS and incubated with $3 \mu \mathrm{M}$ DAPI (Molecular Probes) for $5 \mathrm{~min}$ to label nuclei, followed by a final wash with PBS. Images were acquired using a fluorescent microscope (Nikon).
RhoA activity assay. The RhoA activity assay was performed according to the manufacturer's recommendations (Upstate). Activated (GTP-bound) RhoA was pulled down from whole cell lysates (800 $\mu$ protein/sample) with Rhotekin-conjugated agarose beads for $45 \mathrm{~min}$ at $4{ }^{\circ} \mathrm{C}$. The beads were collected by centrifugation and washed with wash buffer. Activated RhoA was detached from the beads by boiling for $5 \mathrm{~min}$ in Laemmli reducing sample buffer (1x), separated by $10 \%$ SDS-PAGE, and then transferred to a PVDF membrane. After blocking for 30 min at room temperature with 3\% BSA/TBS (bovine serum albumin in Tris-buffered saline), the membranes were incubated with Anti-RhoA antibody overnight at $4{ }^{\circ} \mathrm{C}$. After three washes with TBS, signals were detected using a HRP-conjugated anti-mouse $\operatorname{Ig} \mathrm{G}_{1}$ secondary antibody and an enhanced chemiluminescence detection system (Chemicon).

In vitro differentiation, alkaline phosphatase activity assay and cytochemical assay. The effects of FITC-MSNs uptake on osteogenic differentiation were performed as follows. hMSCs were incubated first with $40 \mu \mathrm{g} / \mathrm{ml}$ of FITC-MSNs for $1 \mathrm{~h}$, followed by a PBS wash and then treatment with osteogenic medium for 1 and 3 days, changing the medium twice weekly. Osteogenic medium consists of $\alpha$-MEM (GIBCO) supplemented with $1 \mu \mathrm{M}$ of dexamethasone, $50 \mu \mathrm{M}$ of $\beta$-glycerolphosphate (Sigma-Aldrich), and $50 \mu \mathrm{g} / \mathrm{ml}$ of ascorbic acid (Sigma-Aldrich). For quantifying alkaline phosphatase activity, cells in 24-well plates were washed with $0.1 \%$ Tween-20/TBS four times; then $400 \mu \mathrm{l}$ of $p$-nitrophenyl phosphate solution (Sigma-Aldrich) was added to each well. After incubation at $37^{\circ} \mathrm{C}$ for $30 \mathrm{~min}$, the reaction was terminated by adding $400 \mu \mathrm{l}$ of $1 \mathrm{~N} \mathrm{NaOH}$. The absorbance at $405 \mathrm{~nm}$ was measured with a microplate reader; and the values were normalized by culture medium-treated samples as 1 . For evaluation of
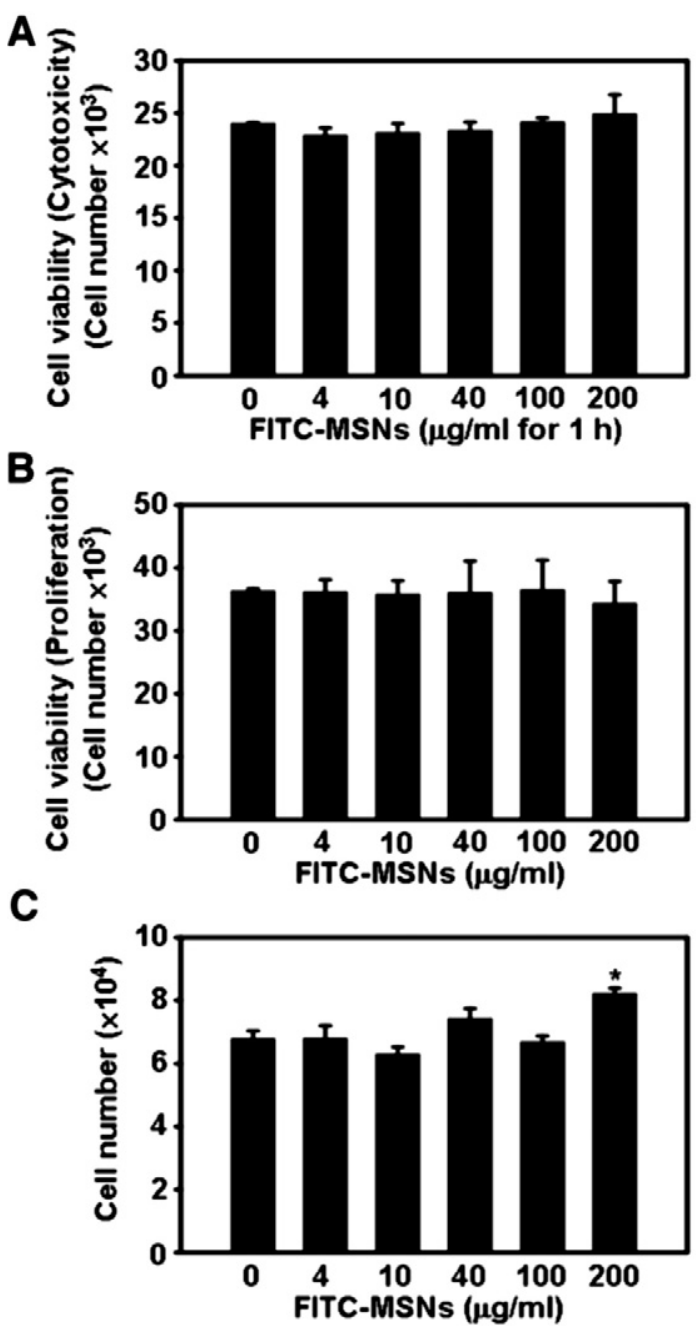

Fig. 2. Effect of FITC-MSNs on cytotoxicity and cell proliferation in hMSCs. (A) Cells treated with FITC-MSNs at the indicated concentrations for $1 \mathrm{~h}$ and then immediately incubated with MTT for cytotoxicity determination. The effect of FITC-MSNs on cell proliferation was examined by MTT assays (B) and hemocytometer counts of trypan blue-excluding cells (C) after hMSCs were allowed to grow in regular growth medium for $24 \mathrm{~h}$ after $1 \mathrm{~h}$ of incubation with the indicated concentrations. Only a slightly significant increase in cell numbers was observed in cells treated with $200 \mu \mathrm{g} / \mathrm{ml}$ of FITC-MSNs. Each point represents the mean \pm SEM of three independent experiments, each performed in four replicate wells. ${ }^{*} \mathrm{P}<0.05$ as compared with control. 
osteogenic differentiation staining, cells were fixed with citrate-buffered acetone and stained with Fast Blue RR Salt (Sigma-Aldrich).

RNA extraction and RT-PCR analysis. Total RNA was extracted from hMSCs using TRIZOL reagent (Invitrogen) according to the manufacturer's instructions. RNA ( $1 \mu \mathrm{g}$ ) was reverse transcribed with Super Script III (Invitrogen) in the presence of oligo-dT primer $(2.5 \mu \mathrm{M})$, dNTP $(0.5 \mathrm{mM})$, and DTT $(0.01 \mathrm{M})$. PCR was performed using primers described in a previous report (Rickard et al., 1996): bone/liver/kidney alkaline phosphatase (5': ACGTGGCTAAGAATGTCATC; 3': CTGGTAGGCGATGTCCTTA). The reaction was performed using a GeneAmp PCR System 9700 (Applied Biosystem) after an initial heating at $95{ }^{\circ} \mathrm{C}$ for $5 \mathrm{~min}$, followed by 35 cycles of denaturation at $95^{\circ} \mathrm{C}$ for $40 \mathrm{~s}$, annealing at $55{ }^{\circ} \mathrm{C}$ for $40 \mathrm{~s}$, and elongation at $72{ }^{\circ} \mathrm{C}$ for $1 \mathrm{~min}$, with an additional 7 -min incubation at $72{ }^{\circ} \mathrm{C}$ after completing the last cycle. The amplified DNA was loaded onto a $1.5 \%$ agarose gel pre-stained with ethidium bromide. After electrophoresis, the DNA bands were photographed under UV light. The gel was photographed using an image analyzer (Gel DOC 2000, BioRad, Hercules, CA). The signal intensity for the alkaline phosphatase (ALP) gene product was normalized to their respective $\beta$-actin expression.

Statistical analysis. Data are presented as the mean \pm standard error of mean (SEM) for the indicated number of separate experiments. Statistical analysis of data was performed with one-way analysis of variance (ANOVA) followed by Dunnett's 2 sided $t$-test. $P$-values less than 0.05 were considered significant.

\section{Results}

Preparation and characterization of FITC-MSNs

Fluorescein isothiocyanate (FITC) attached mesoporous silica nanoparticles (FITC-MSNs) with well-ordered hexagonal pore structure were synthesized under dilute tetraethyl orthosilicate (TEOS) and low surfactant conditions with aqueous ammonia as a catalyst in a two-step preparation method as described in a previous study (Lin et al., 2005). TEM of FITC-MSNs is shown in Fig. 1A which indicates that the nanoparticles were about $110 \mathrm{~nm}$ in size and were largely discrete. High-resolution TEM (HRTEM, Fig. 1B) shows the orderedporous nature. The mesostructure of the samples was examined by XRD (Fig. 1C). XRD patterns exhibited three hexagonal characteristic reflection peaks (100), (110), and (200) reflecting on the ordered pore structure. The $d_{100}$ spacing of FITC-MSN was $3.97 \mathrm{~nm}$. The physical properties of mesoporous silica nanoparticles (MSNs) were analyzed by $\mathrm{N}_{2}$ adsorption-desorption isotherms (Figs. 1D and E). The nanoparticles had high surface area $\left(1172 \mathrm{~m}^{2} \mathrm{~g}^{-1}\right)$, high pore volume $\left(0.69 \mathrm{~cm}^{3} \mathrm{~g}^{-1}\right)$, and uniform BJH pore size distribution $(2.6 \mathrm{~nm})$. The FITC-MSNs exhibited characteristic type IV isotherm; and no hysteresis loop was observed. In addition, the secondary adsorption step at $0.95 P / P_{0}$ was attributed to the textural pores formed by the closely packed nanoparticles. The average hydrodynamic diameter of MSN was measured using dynamic light scattering (DLS) (Fig. 1F). A narrow size distribution was observed, implying that these particles are discrete and near-uniform size. Our previous study (Lin et al., 2005) described FITC-MSNs in more detail.

\section{The biocompatibility and cellular uptake of FITC-MSNs}

After treatment with $0-200 \mu \mathrm{g} / \mathrm{ml}$ of FITC-MSNs for $1 \mathrm{~h}$, hMSCs were either processed immediately with MTT incubation for acute cytotoxicity determination (Fig. 2A) or followed by 24-hour incubation in cell culture medium for cell proliferation by MTT reduction (Fig. 2B) and trypan blue exclusion assays (Fig. 2C). As shown in Fig. 2, no evidence of cytotoxicity was observed at $1 \mathrm{~h}$ treatment of FITCMSNs, nor was the cell proliferation inhibited.

Since FITC-MSNs contained the fluorescent dye, the degree of MSNs uptake was quantified with flow cytometry by determining the green fluorescence emitted from FITC-MSN-treated cells. The logarithmic fluorescence intensity of untreated cells was set between $10^{\circ}$
A

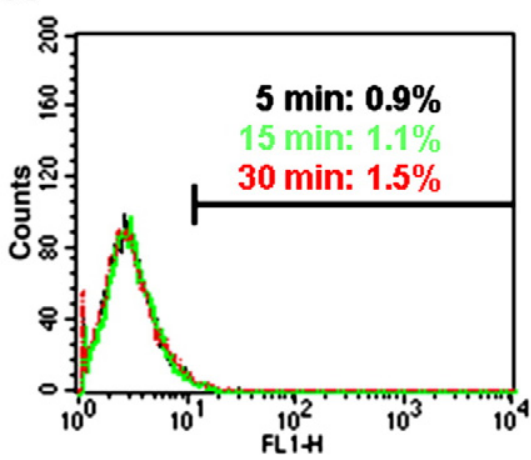

C

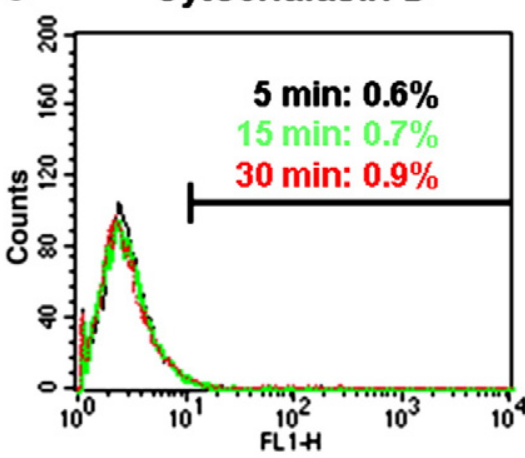

B

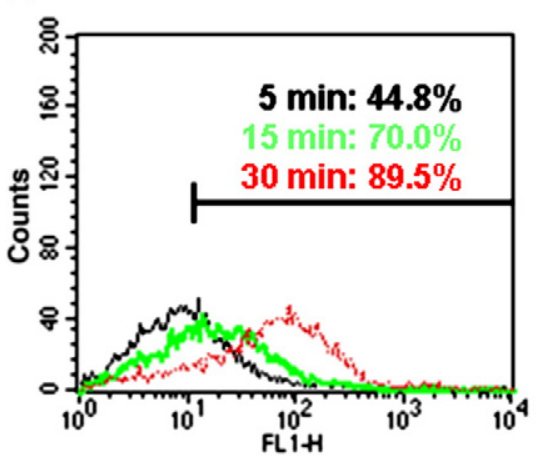

FITC-MSNs

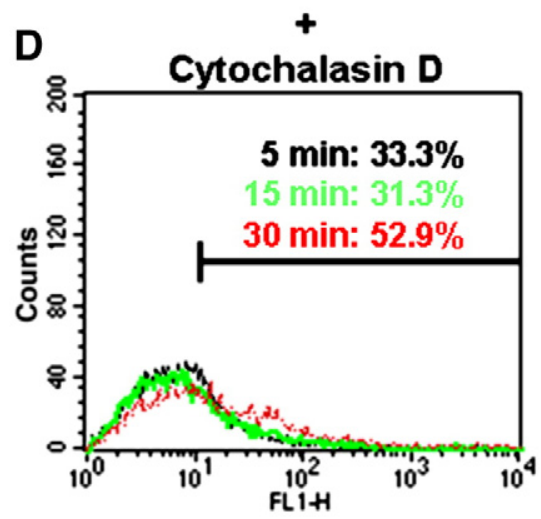

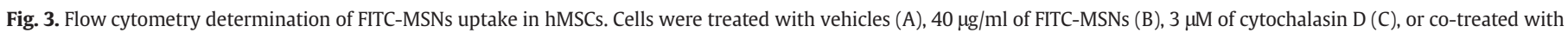
FITC-MSNs and cytochalasin D for 5, 15, and $30 \mathrm{~min}$. The number of positively labeled cells was represented as the percentage of total counting cells in each panel. 
and $10^{1}$, hence cells that exhibited green fluorescence intensity higher than $10^{1}$ were taken as positively labeled cells with FITC-MSNs. After incubation with FITC-MSNs for 5, 15, and $30 \mathrm{~min}$ at the same concentration as used in biocompatibility assay (in the following experiments, treating cells with $40 \mu \mathrm{g} / \mathrm{ml}$ of FITC-MSNs was employed), hMSCs were labeled in a time-dependent manner. In the 30-min incubation, most cells were labeled. Typical flow cytometry plots are shown in Fig. 3, with the percentages of positively labeled cells noted in each panel. The internalization of FITC-MSNs was significantly inhibited by the co-treatment with an actin polymerization inhibitor, cytochalasin D (Figs. 3B and D).

\section{Effects of the uptake of FITC-MSNs on actin organization}

Control cells (treated with vehicles) exhibited strong actin stress fiber staining (Fig. 4A). Viable nuclei (blue) are visible in each condition by DAPI stain. Cells treated with FITC-MSNs showed prominent well-organized actin stress fibers, were observed as compared with control cells (Figs. 4A and B), suggesting the internalization of FITC-MSNs can enhance actin polymerization. To further explore the crosstalk between internalization of FITC-MSNs and actin polymerization induction, cells were pre-incubated with cytochalasin D for 5 min to disrupt actin filaments and then treated with FITCMSNs. As shown in Fig. 4C, cells treated with cytochalasin D were much more rounded and lacked stress fibers. Interestingly, the incubation of FITC-MSNs caused a dramatic increase in actin polymerization and extended cellular morphology in cytochalasin D-treated cells (Figs. 4C and D); the stress fiber formation and the recovery of cell shape, however, were not fully restored to control levels
A

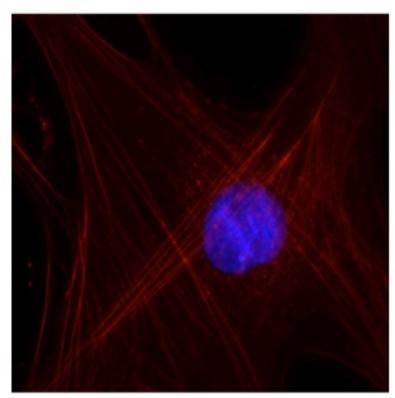

B

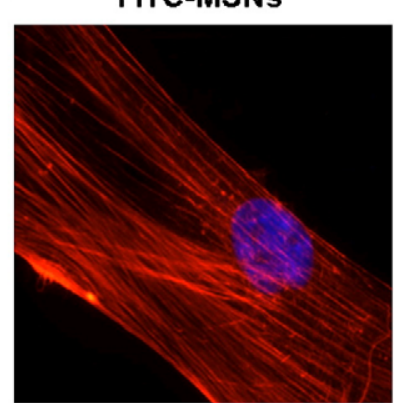

\section{Cytochalasin D}

D

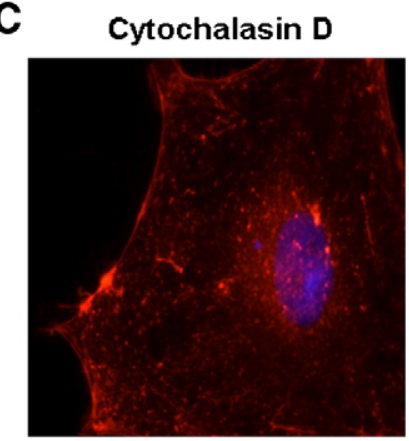

A

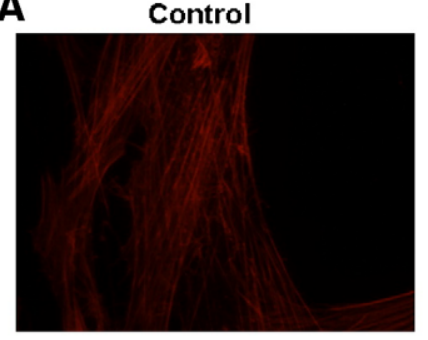

C
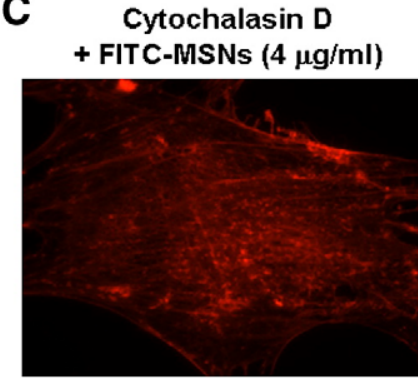

E

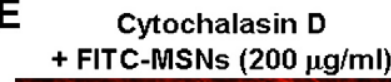

B

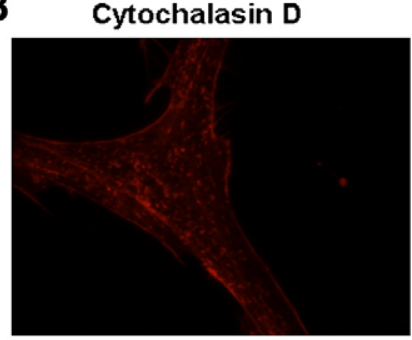

D
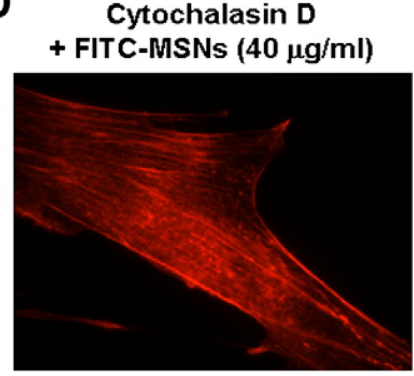

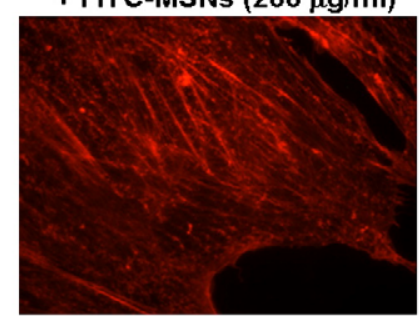

Fig. 5. Dose-dependent effect of FITC-MSNs on cytochalasin D-mediated actin depolymerization. Cells were pre-treated with vehicle (A) or $1 \mu \mathrm{M}$ of cytochalasin $\mathrm{D}$ (B to $\mathrm{E}$ ) for $5 \mathrm{~min}$, followed by a wash and then incubation with 0 (A and B), 4 (C), 40 (D) and 200 (E) $\mu \mathrm{g} / \mathrm{ml}$ of FITC-MSNs for $20 \mathrm{~min}$. Filamentous actin (red) was stained with rhodamine-phalloidin. Images were acquired using fluorescent microscopy under a $\times 100$ objective. Images are representative of three separate experiments.

(Fig. 4A). Moreover, the regressive effect of FITC-MSNs on cytochalasin D-mediated actin depolymerization was dose-dependent (Fig. 5).

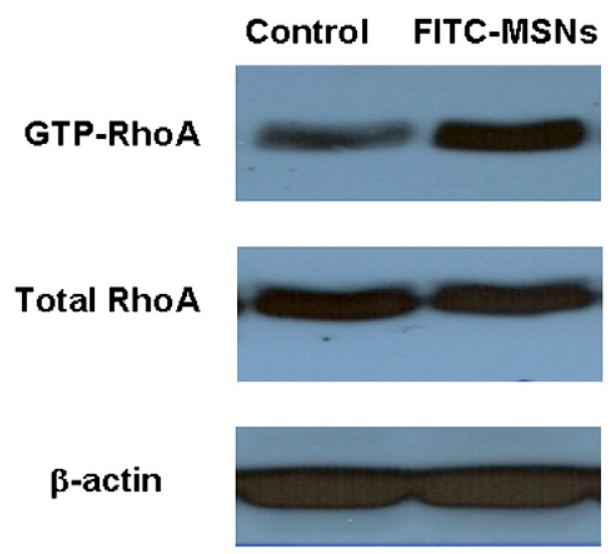

Fig. 6. RhoA activity assay. hMSCs were treated with vehicle or $40 \mu \mathrm{g} / \mathrm{ml}$ of FITC-MSNs for $15 \mathrm{~min}$. Whole cell protein was extracted for immunoblot analysis of total RhoA expression and $\beta$-actin as protein loading control. Activated (GTP-bound) RhoA was pulled down from total protein $(800 \mu \mathrm{g})$ using Rhotekin-conjugated agarose beads. The image is a representative of three independent experiments, each performed in duplicate. 
Activation of RhoA by FITC-MSNs

Total RhoA protein expression was confirmed by Western blot analysis. There was no significant change in total RhoA protein expression between control cells and FITC-MSN-treated cells. In contrast, we detected an obvious induction in the activated, GTP-bound form of RhoA in FITC-MSN-treated cells (Fig. 6).

\section{Effects of FITC-MSNs on the early stage of osteogenic differentiation}

Qualitative staining of alkaline phosphatase (ALP) expression by Fast Blue showed an apparent osteogenesis of hMSCs after incubation with osteogenic medium for 3 days (Fig. 7D vs. A). In contrast, there was no detectable induction of osteogenesis in hMSCs after 1-day incubation (Fig. 7B vs. A). However, FITC-MSNs did not significantly induce osteogenesis in the early stages (osteogenic induction for no more than 3 days) of the differentiation process (Fig. 7C vs. B; Fig. 7E vs. D). Similar to what had been the case with the qualitative assay of osteogenesis, quantifying the activity of ALP revealed an obvious osteogenic differentiation occurred at 3-day incubation and that untreated and FITC-MSN-treated cells did not react in a significantly different way to osteogenesis (Fig. 8A). However, when the effect of FITC-MSNs on ALP gene expression was further examined by reverse transcriptase polymerase chain reaction (RT-PCR), we found that the FITC-MSN-treated hMSCs exhibited significantly higher ALP mRNA

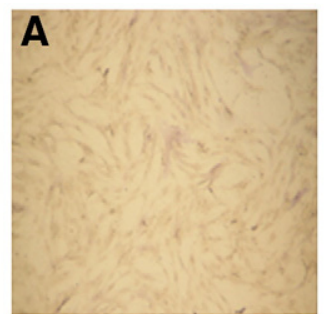

\section{Control medium}

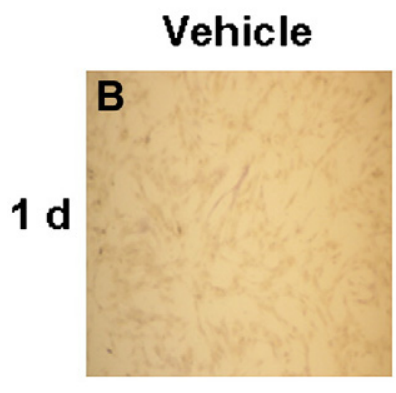

\section{FITC-MSNs}
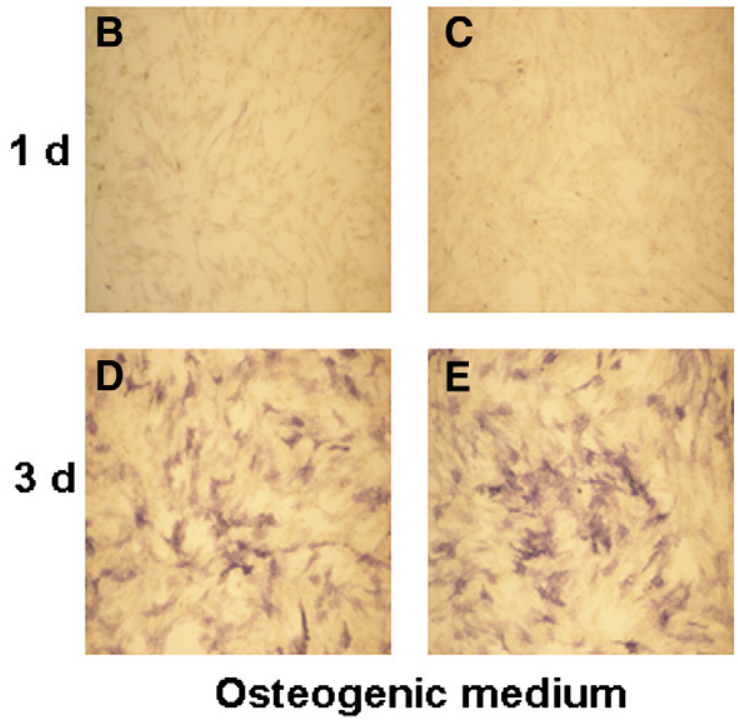

Fig. 7. The effect of FITC-MSNs on short-term osteogenic differentiation in hMSCs. Cells were incubated without (B and D) or with (C and E) $40 \mu \mathrm{g} / \mathrm{ml}$ of FITC-MSNs for $1 \mathrm{~h}$, followed by a wash and then incubation with osteogenic medium for 1 (B and $C$ ) and 3 (D and E) days. Osteogenic differentiation was demonstrated by Fast Blue staining for detecting alkaline phosphatase expression. A detectable stain occurred in cells incubated with osteogenic medium for 3 days but not for 1 day. Cells incubated with culture medium were for the un-differentiation control (A).
A

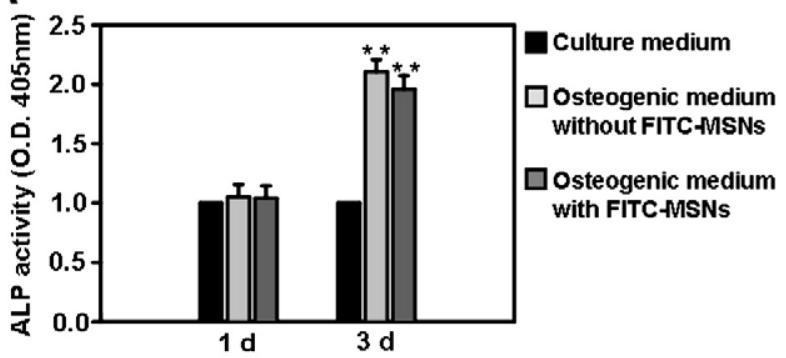

B

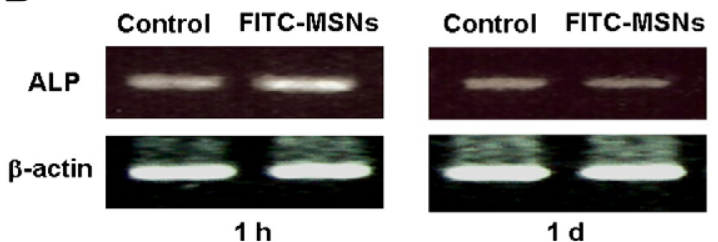

C

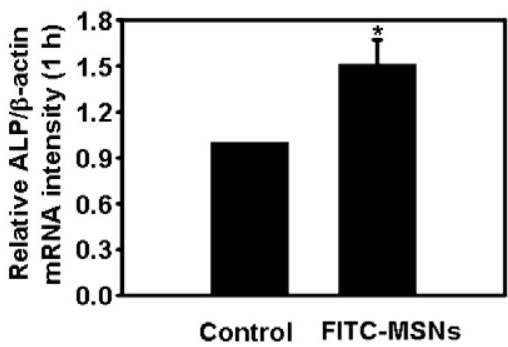

Fig. 8. The effect of the internalization of FITC-MSNs on protein activity and gene expression of alkaline phosphatase (ALP). (A) hMSCs were treated with or without $40 \mu \mathrm{g} / \mathrm{ml}$ of FITC-MSNs for $1 \mathrm{~h}$, followed by a wash and then incubation with osteogenic medium for 1 and 3 days. Cells incubated with culture medium were used for un-differentiation control. Fold of ALP activity induction was obtained by dividing the absorbance values by that of the un-differentiation control. Data are expressed as mean \pm SEM of three to four independent experiments. ${ }^{* *} P<0.001$ as compared with the un-differentiation control. (B) Cells were treated with or without $40 \mu \mathrm{g} / \mathrm{ml}$ of FITC-MSNs for $1 \mathrm{~h}$, followed by a wash and then total RNA was extracted at $1 \mathrm{~h}$ and 1 day after osteogenic induction. Expression of ALP was analyzed by RT-PCR. Expression of $\beta$-actin was shown as RNA loading control. (C) The signal intensities of ALP and $\beta$-actin $(1 \mathrm{~h})$ were quantified using an image analyzer; and the changes in the signal intensity of ALP relative to $\beta$-actin were calculated and expressed as mean \pm SEM of four independent experiments. $* P<0.01$ as compared with control.

levels than control cells after 1-hour osteogenic induction (Figs. 8B and $C$ ). The signal intensities of ALP and $\beta$-actin were quantified using an image analyzer; see Fig. $8 \mathrm{C}$ for the changes in the signal intensity of ALP relative to $\beta$-actin. The induction of ALP gene expression by FITCMSNs was absent in cells after 1-day incubation.

\section{Discussion}

Nanoparticles may interfere with biological functions or induce the cytotoxicity in several ways, many studies have shown. These ways include but are not limited to the following three examples. (1) Obviously, the inherent chemical composition of some nanoparticles can be toxic. Derfus at al. reported that the concentration of the free cadmium ions $\left(\mathrm{Cd}^{2+}\right)$ released from CdSe particles in aqueous solution directly correlated with cell death in primary hepatocytes (Derfus et al., 2004). Tests of the cytotoxicity of different carbon nanomaterials including single-wall nanotubes, multi-wall nanotubes, and fullerene $\left(C_{60}\right)$ - revealed that single-wall nanotubes significantly impaired phagocytosis and induced cell death in alveolar macrophages (Jia et al., 2005). In addition, Hussain et al. demonstrated that silver nanoparticle were more highly toxic than other tested metal/metal oxide nanoparticles in rat liver derived cell line (BRL 3A); they suggested that the cytotoxicity of silver is likely mediated through oxidative stress (Hussain et al., 2005). (2) The chemical and physical properties (e.g., 
particle size and surface coating material) of nanoparticles might contribute to the cytotoxic effects in important ways. For example, although chitosan molecules and nanoparticles exhibited comparable cytotoxicity in A549 cells, the cytotoxicity of both chitosan entities was attenuated by decreasing the deacetylation degree but was less affected by a lowering in molecular weight (Huang et al., 2004). Gupta and Gupta reported that modifying the surface of superparamagnetic iron oxide nanoparticles with pullulan, a nonionic polysaccharide, reduced the cytotoxicity of the nanoparticles in telomerase-immortalized primary human fibroblasts (hTERT-BJ1) (Gupta and Gupta, 2005). Yin et al. demonstrated that nickel ferrite nanoparticles coated with oleic acid as a monomer were not cytotoxic and that the particle size was not a significant factor on cytotoxicity; large particles, however, had a larger cytotoxic effect than smaller particles when one or two layers of oleic acid were deposited on particle surface (Yin et al., 2005). Moreover, for quantum dots, an appropriate coating can render particles nontoxic (Derfus et al., 2004). (3) There might be effects caused by the shape of the particles and the interaction manner between cells and particles themselves. For instance, it has been reported that carbon nanotubes can impale cells like needles (Lam et al., 2004; Warheit et al., 2004). Furthermore, the location of particles will influence their cytotoxicity. Particles that are internalized into cells caused different cytotoxicity than particles mantling the cell membrane (Kirchner et al., 2005). These cytotoxicity studies on nanomaterials, however, have focused mainly on the cellular functional assay rather than highlighted the particularities of biomedical application of different nanoparticles.

Tracking the distribution of stem cells is crucial to their therapeutic use. For stem cell tracking, the biological effects of the internalized nanoparticles surely have priority over other considerations, including the cellular-labeling efficiency. Although a number of studies (Lewin et al., 2000; Bulte et al., 2001; Rudelius et al., 2003; Huang et al., 2005) indicate that internalized nanoparticles have no apparent deleterious effects on the morphology, cell proliferation, cell viability and differentiation efficiency in stem cells, detailed characterizations of cellular activities have not been offered. On the other hand, recent studies have examined deleterious effects of the internalized nanoparticles on stem cells. For example, in human bone marrow mesenchymal stem cells (hMSCs), Kostura et al. demonstrated that poly-L-lysine (PLL)coated Feridex, an FDA-approved SPIO formulation in an off-label application, markedly diminished the chondrogenesis but not cell viability, proliferation, and adipogenic and osteogenic differentiation. The inhibition of chondrogenic activity was mediated by the Feridex, rather than by the transfection agent (PLL) (Kostura et al., 2004). Additionally, Hsieh et al. showed that the introduction of quantum dots significantly suppressed the expression of osteocyte-specific marker genes and the activity of alkaline phosphatase (ALP) but not the cell growth or the morphology and mineralization of hMSCs (Hsieh et al., 2006). The deleterious effects, however, were suggested to have been derived from the chemical composite of the nanoparticles, as in other cytotoxicity studies such as those mentioned above; and the molecular signal pathway, which underlines the deleterious effects, was not explored.

Although our previous study showed that FITC-MSNs were highly biocompatible and were able to internalize into hMSCs efficiently (Huang et al., 2005), in the present report we repeated the cellular uptake study and cytotoxicity assay of FITC-MSNs. As expected and evidenced previously, exposure to FITC-MSNs resulted in no significantly reduced cell viability in acute cytotoxicity assay and long-term cellular proliferation (Fig. 2). The flow cytometry data (Figs. 3A and B) shows that FITC-MSNs internalized into hMSCs in seconds demonstrated highly efficient cellular labeling consistent with our previous report (Huang et al., 2005). Although FITC-MSN treatment showed the material to be highly biocompatible, the intense dependence of the uptake of FITC-MSNs on well-polymerized actin (Figs. 3B and D) suggested an interaction between actin dynamics and the internalized FITC-MSNs. In addition, it is inevitable to suspect the biocompatibility of so many FITC-MSNs inside cells resulting from their high efficiency of internalization; therefore, we examined the effects of the internalization of FITC-MSNs on actin dynamics.

To determine the effects of cellular uptake of FITC-MSNs on actin organization, hMSCs were fixed and stained with rhodamine-phalloidin to label actin stress fibers. Moreover, an actin-disrupting reagent cytochalasin D was added to examine the aptitude of FITC-MSNs for regulating actin dynamics. As shown in Figs. 4 and 5, there was an internalization- and dose-dependent up-regulation of actin cytoskeletal organization following the treatment of FITC-MSNs in hMSCs.

Activation of the small GTPase, RhoA, is required for stress fiber formation (Hall, 1998). Therefore, we examined the activation state of RhoA in hMSCs after FITC-MSNs treatment. Western blot analysis showed the induction of increased RhoA activity along with actin polymerization by the internalization of FITC-MSNs in hMSCs (Fig. 6). RhoA-mediated actin cytoskeletal tension plays a decisive role in the lineage of hMSCs (Sordella et al., 2003; McBeath et al., 2004; Meyers et al., 2005). Cell shape, actin cytoskeletal tension, and RhoA activation have recently been suggested to lead hMSCs into osteogenic differentiation (McBeath et al., 2004). However, no significant difference of osteogenesis was observed in FITC-MSN-treated cells as compared to cells that were not exposed to the nanoparticles in our previous reports (Huang et al., 2005). One possible explanation for the inability of RhoA activation/actin organization to induce osteogenesis may be that the uptake of FITC-MSNs for efficient cellular labeling required only a short incubation time (less than $1 \mathrm{~h}$, Fig. 3A); hence FITC-MSN-mediated cellular responses were too transient to affect a long-term osteogenesis process. Thus, we investigated the impact of the uptake of FITC-MSNs on the initial stage of osteogenic differentiation.

To achieve the full response of osteogenesis, hMSCs are usually incubated with osteogenic medium for several days to weeks. In the present study, we observed the osteogenic differentiation status after hMSCs were incubated with osteogenic medium for $1 \mathrm{~h}, 1$ day, and 3 days. Although FITC-MSNs did not affect the regular osteogenesis of hMSCs (Figs. 7 and 8A), our data showed that FITC-MSNs indeed induced a significant but transient osteogenic signal in hMSCs (Figs. 8B and C).

In conclusion, despite the many benefits of nanotechnology in biomedicine, a number of studies indicate that certain nanoparticles may cause adverse effects. Although some studies have suggested that nanoparticles could be applied in biomedicine due to their biosafety, a standardization of cytotoxicity evaluation is still required before we can claim the material biocompatible. In the present study, we have explored the biocompatibility of MCM-41-type mesoporous silica nanoparticles as a stem cell labeling agent in distinct and deeper views of osteogenic differentiation. Although cell viability, cell proliferation, and regular osteogenic differentiation of hMSCs are not affected by the internalized MSNs, the actin polymerization, RhoA activity, and induced expression of the osteocyte-specific marker gene, alkaline phosphatase, are significantly upregulated fleetingly. The possibility of side effects of MSNs in applications in other biological fields, however, should not be ignored. Therefore, based on the present findings, we suggest that the effects of nanoparticles on diverse aspects of cellular activities should be carefully evaluated even though the nanoparticles are generally considered as biocompatible.

\section{Acknowledgments}

This work was supported by grants from the National Science Council (NSC95-2320-B-400-013) of Taiwan and the National Health Research Institutes, (Grant NM-096-PP-02 and NM-096-PP-09), Taiwan.

\section{References}

Bellocq, N.C., Pun, S.H., Jensen, G.S., Davis, M.E., 2003. Transferrin-containing, cyclodextrin polymer-based particles for tumor-targeted gene delivery. Bioconjug. Chem. 14, 1122-1132. 
Bulte, J.W.M., Ma, L.D., Magin, R.L., Kamman, R.L., Hulstaert, C.E., Go, K.G., The, T.H., de Leij, L., 1993. Selective MR imaging of labeled human peripheral blood mononuclear cells by liposome mediated incorporation of dextran-magnetite particles. Magn. Reson. Med. 29, 32-37.

Bulte, J.W.M., Douglas, T., Witwer, B., Zhang, S.-C., Strable, E., Lewis, B.K., Zywicke, H. Miller, B., van Gelderen, P., Moskowitz, B.M., Duncan, I.D., Frank, J.A., 2001. Magnetodendrimers allow endosomal magnetic labeling and in vivo tracking of stem cells. Nat. Biotechnol. 19, 1141-1147.

Chung, T.H., Wu, S.H., Yao, M., Lu, C.W., Lin, Y.S., Hung, Y., Mou, C.Y., Chen, Y.C., Huang, D.M., 2007. The effect of surface charge on the uptake and biological function of mesoporous silica nanoparticles in 3T3-L1 cells and human mesenchymal stem cells. Biomaterials $28,2959-2966$.

Colvin, V.L., 2003. The potential environmental impact of engineered nanomaterials. Nat. Biotechnol. 21, 1166-1170.

Derfus, A.M., Chan, W.C.W., Bhatia, S.N., 2004. Probing the cytotoxicity of semiconductor quantum dots. Nano Lett. 4, 11-18.

Giles, J., 2004. Size matters when it comes to safety, report warns. Nature 430, 599.

Giri, S., Trewyn, B.G., Stellmaker, M.P., Lin, V.S.-Y., 2005. Stimuli-responsive controlledrelease delivery system based on mesoporous silica nanorods capped with magnetic nanoparticles. Angew. Chem. Int. Ed. Engl. 44, 5038-5044.

Gupta, A.K., Gupta, M., 2005. Cytotoxicity suppression and cellular uptake enhancement of surface modified magnetic nanoparticles. Biomaterials 26, 1565-1573.

Hall, A., 1998. Rho GTPase and the cytoskeleton. Science 279, 509-514.

Hoet, P.H.M., Nemmar, A., Nemery, B., 2004. Health impact of nanomaterials? Nat. Biotechnol. 22, 19 .

Hsieh, S.-C., Wang, F.-F., Lin, C.-S., Chen, Y.-J., Hung, S.-C., Wang, Y.-J., 2006. The inhibition of osteogenesis with human bone marrow mesenchymal stem cells by CdSe/ZnS quantum dot labels. Biomaterials 27, 1656-1664.

Huang, D.-M., Hung, Y., Ko, B.-S., Hsu, S.-C., Chen, W.-H., Chien, C.-L., Chien, C.-L., Tsai, C.-P., Kuo, C.-T., Kang, J.-C., Yang, C.-S., Mou, C.-Y., Chen, Y.-C., 2005. Highly efficient cellular labeling of mesoporous nanoparticles in human mesenchymal stem cells: implication for stem cell tracking. FASEB J. 19, 2014-2016.

Huang, M., Khor, E., Lim, L.-Y., 2004. Uptake and cytotoxicity of chitosan molecules and nanoparticles: effects of molecular weight and degree of deacetylation. Pharm. Res. 21, 344-353.

Hussain, S.M., Hess, K.L., Gearhart, J.M., Geiss, K.T., Schlager, J.J., 2005. In vitro toxicity of nanoparticles in BRL 3A rat liver cells. Toxicol. in Vitro 19, 975-983.

Jia, G., Wang, H., Yan, L., Wang, X., Pei, R., Yan, T., Zhao, Y., Guo, X., 2005. Cytotoxicity of carbon nanomaterials: single-wall nanotube, multi-wall nanotube, and fullerene. Environ. Sci. Technol. 39, 1378-1383.

Kirchner, C., Liedl, T., Kudera, S., Pellegrino, T., Javier, A.M., Gaub, H.E., Stolzle, S., Fertig N., Parak, W.J., 2005. Cytotoxicity of colloidal CdSe and CdSe/ZnS nanoparticles. Nano Lett. 5, 331-338.

Kostura, L., Kraitchman, D.L., Mackay, A.M., Pittenger, M.F., Bulte, J.W.M., 2004. Feridex labeling of mesenchymal stem cells inhibits chondrogenesis but not adipogenesis or osteogenesis. NMR Biomed. 17, 513-517.
Lai, C.-Y., Trewyn, B.G., Jeftinija, D.M., Jeftinija, K., Xu, S., Jeftinija, S., Lin, V.S.-Y., 2003. A mesoporous silica nanosphere-based carrier system with chemically removable CdS nanoparticle caps for stimuli-responsive controlled release of neurotransmitters and drug molecules. J. Am. Chem. Soc. 125, 4451-4459.

Lam, C.-W., James, J.T., McCluskey, R., Hunter, R.L., 2004. Pulmonary toxicity of singlewall carbon nanotubes in mice 7 and 90 days after intratracheal instillation. Toxicol. Sci. 77, 126-134.

Larson, D.R. Zipfel, W.R., Williams, R.M., Clark, S.W., Bruchez, M.P., Wise, F.W., Webb, W.W., 2003. Water-soluble quantum dots for mutiphoton fluorescence imaging in vivo. Science 300, 1434-1436.

Lewin, M., Carlesso, N., Tung, C.-H., Tang, X.-W., Cory, D., Scadden, D.T., Weissleder, R. 2000. Tat peptide-derivatized magnetic nanoparticles allow in vivo tracking and recovery of progenitor cells. Nat. Biotechnol. 18, 410-414.

Lin, Y.-S., Tsai, C.-P., Huang, H.-Y., Kuo, C.-T., Hung, Y., Huang, D.-M., Chen, Y.-C., Mou, C.-Y., 2005. Well-ordered mesoporous silica nanoparticles as cell markers. Chem. Mater. 17, 4570-4573.

McBeath, R., Pirone, D.M., Nelson, C.M., Bhadriraju, K., Chen, C.S., 2004. Cell shape, cytoskeleton tension, and RhoA regulate stem cell lineage commitment. Dev. Cell 6, 483-495.

Meyers, V.E., Zayzafoon, M., Douglas, J.T., McDonald, J.M., 2005. RhoA and cytoskeletal disruption mediate reduced osteoblastogenesis and enhanced adipogenesis of human mesenchymal stem cells in modeled microgravity. J. Bone Miner. Res. 20, 1858-1866.

Ren, X.-D., Kiosses, W.B., Schwartz, M.A., 1999. Regulation of the small GTP-binding protein Rho by cell adhesion and the cytoskeleton. EMBO J. 18, 578-585.

Rickard, D.J., Kassem, M., Hefferan, T.E., Sarkar, G., Spelsberg, T.C., Riggs, B.L., 1996. Isolation and characterization of osteoblasts precursor cells from human bone marrow. J. Bone Miner. Res. 11, 312-324.

Roco, M.C., 2003. Nanotechnology: convergence with modern biology and medicine. Curr. Opin. Biotechnol. 14, 337-346.

Rudelius, M., Daldrup-Link, H.E., Heinzmann, U., Piontek, G., Settles, M., Link, T.M., Schlegel, J., 2003. Highly efficient paramagnetic labelling of embryonic and neuronal stem cells. Eur. Nucl. Med. Mol. Imaging 30, 1038-1044.

Sahoo, S.K., Labhasetwar, V., 2003. Nanotech approaches to drug delivery and imaging. Drug Discov. Today 8, 1112-1120.

Slowing, I., Trewyn, B.G., Lin, V.S.-Y., 2006. Effect of surface functionalization of MCM41-type mesoporous silica nanoparticles on the endocytosis by human cancer cells. J. Am. Chem. Soc. 128, 14792-14793.

Sordella, R., Jiang, W., Chen, G.-C., Curto, M., Settleman, J., 2003. Modulation of Rho GTPase signalling regulates a switch between adipogenesis and myogenesis. Cell 113, 147-158.

Warheit, D.B., Laurence, B.R., Reed, K.L., Roach, D.H., Reynolds, G.A.M., Webb, T.R., 2004. Comparative pulmonary toxicity assessment of single-wall carbon nanotubes in rats. Toxicol. Sci. 77, 117-125.

Wilkinson, J.M., 2003. Nanotechnology applications in medicine. Med. Device Technol. $14,29-31$.

Yin, H., Too, H.P., Chow, G.M., 2005. The effects of particle size and surface coating on the cytotoxicity of nickel ferrite. Biomaterials 26, 5818-5826. 